\title{
A BERNSTEIN TYPE RESULT FOR SPECIAL LAGRANGIAN SUBMANIFOLDS
}

\author{
Mao-Pei Tsui and Mu-Tao Wang
}

\begin{abstract}
Let $\Sigma$ be a complete minimal Lagrangian submanifold of $\mathbb{C}^{n}$. We identify several regions in the Grassmannian of Lagrangian subspaces so that whenever the image of the Gauss map of $\Sigma$ lies in one of these regions, then $\Sigma$ is an affine space.
\end{abstract}

\section{Introduction}

The well-known Bernstein theorem states any complete minimal surface that can be written as the graph of a function on $\mathbb{R}^{2}$ must be a plane. This type of result has been generalized in higher dimension and codimension under various conditions. See [2] and the reference therein for the codimension one case and [1], [3], [4] and [7] for higher codimension case. In this note, we prove a Bernstein type result for complete minimal Lagrangian submanifolds of $\mathbb{C}^{n}$. We remark that Jost-Xin [8] obtained similar results from a somewhat different approach.

Recall a submanifold $\Sigma$ of $\mathbb{C}^{n}$ is called Lagrangian if the Kähler form $\sum_{i=1}^{n}$ $d x^{i} \wedge d y^{i}$ restricts to zero on $\Sigma$. If $\Sigma$ happens to be the graph of a vector-valued function from a Lagrangian subspace $L$ to its complement $L^{\perp}$ in $\mathbb{C}^{n}$. Rotating $\mathbb{C}^{n}$ by a element in $U(n)$, we may assume $L$ is the $x^{i}$ subspace and $L^{\perp}$ is the $y^{i}$ subspace. In this case, there exists a smooth function $F: \mathbb{R}^{n} \rightarrow \mathbb{R}$ such that $\Sigma$ is defined by the gradient of $F, \nabla F$. The minimal Lagrangian equation can be written in terms of $F$.

$$
\operatorname{Im}\left(\operatorname{det}\left(e^{i \theta}(I+i \operatorname{Hess}(F))\right)\right)=0
$$

where $I=$ identity matrix, Hess $F=\left(\frac{\partial^{2} F}{\partial x^{i} \partial x^{j}}\right)$ and $\theta$ is a constant.

Such minimal submanifolds were studied by Harvey and Lawson [6] in the context of calibrated geometry. In fact, they are calibrated by $n$ forms of the type $\operatorname{Re}\left(e^{i \theta} d z^{1} \wedge \cdots \wedge d z^{n}\right)$ for some constant $\theta$. They are usually referred as special Lagrangian submanifold (SLg) in literature in a more general sense. Recently, Strominger-Yau-Zaslow [9] proposed a geometric construction of mirror manifold through special Lagrangian tori fibration.

Received October 25, 2001.

Revised version received Jan 21, 2002.

The second author is supported in part by N.S.F. grant DMS 0104163. 
In terms of (1.1), the Bernstein type question is to determine under what conditions an entire solution $F$ becomes a quadratic polynomial.

The results in this paper impose conditions on the image of the Gauss map of $\Sigma$. Recall the set of all Lagrangian subspaces of $\mathbb{C}^{n}$ is parametrized by the Lagrangian Grassmannian $U(n) / S O(n)$. The Gauss map of a Lagrangian submanifold $\gamma: \Sigma \mapsto U(n) / S O(n)$ assigns to each $x \in \Sigma$ the tangent space at $x$, $T_{x} \Sigma$.

A particular subset of the Lagrangian Grassmannian consists of the graphs of any symmetric linear transformation from $\mathbb{R}^{n}$ to $\mathbb{R}^{n}$. These can be considered as Lagrangians defined by the gradient of quadratic polynomials on $\mathbb{R}^{n}$.

For any $K>0$, let $\mathfrak{B}_{K}$ denote the subset of the Lagrangian Grassmannian consisting of graphs of symmetric linear transformations $L: \mathbb{R}^{n} \mapsto \mathbb{R}^{n}$ with eigenvalues $\left|\lambda_{i}\right| \leq K$ for each $i$. We remark that if the Gauss map of $\Sigma$ lies in $\mathfrak{B}_{K}$ then $\Sigma$ is the graph of $f: \mathbb{R}^{n} \mapsto \mathbb{R}^{n}$ with uniformly bounded $|d f|$.

Theorem A. Denote by $\Xi$ the subset of the Lagrangian Grassmannian consisting of graphs of symmetric linear transformations $L: \mathbb{R}^{n} \mapsto \mathbb{R}^{n}$ with eigenvalues $\lambda_{i} \lambda_{j} \geq-1$ for any $i, j$. Let $\Sigma$ be a complete minimal Lagrangian submanifold of $\mathbb{C}^{n}$. Suppose there exists an element $g \in U(n)$ such that the image of the Gauss map of $g(\Sigma)$ lies in $\Xi \cap \mathfrak{B}_{K}$, then $\Sigma$ is an affine space.

We remark that the gradient of $g(\Sigma)$ is not necessarily bounded.

Indeed, the most general theorem of this type is the following.

Let $\mathfrak{M}$ be the set of graphs of all symmetric linear transformation $L: \mathbb{R}^{n} \mapsto \mathbb{R}^{n}$ whose eigenvalues $\left(\lambda_{i}\right)$ satisfy the following two conditions:

1.

$$
F\left(h_{i j k}\right)=\sum_{i, j, k} h_{i j k}^{2}+\sum_{k, i} \lambda_{i}^{2} h_{i i k}^{2}+2 \sum_{k, i<j} \lambda_{i} \lambda_{j} h_{i j k}^{2} \geq 0
$$

2 .

for any trace-free symmetric three tensor $h_{i j k}$.

$$
F\left(h_{i j k}\right)=0
$$

if and only if $h_{i j k}=0$ for all $i, j, k$.

Here $h_{i j k}$ is any element in $\otimes^{3} \mathbb{R}^{n}$ that is symmetric in $i, j$ and $k$. $h_{i j k}$ being trace-free means $\sum_{i=1}^{n} h_{i i k}=0$ for any $k$. In fact, $h_{i j k}$ corresponds to the second fundamental form of a Lagrangian submanifold. The trace-free condition corresponds to vanishing mean curvature vector. It is clear that $\Xi$ is a subset of $\mathfrak{M}$.

Theorem B. The conclusion for Theorem A holds for $\mathfrak{M}_{K}$, the subset of the Lagrangian Grassmannian consisting of graphs of symmetric linear transformations in $\mathfrak{M} \cap \mathfrak{B}_{K}$.

These theorems are proved by maximum principle. When $\Sigma$ is the graph over a Lagrangian subspace $L$, we calculate the Laplacian of $\ln * \Omega$ where $* \Omega$ is the Jacobian of the projection from $\Sigma$ to $L$. This is a positive function and when 
the Gauss map of $\Sigma$ satisfies the above conditions it is indeed superharmonic. The parabolic version of this equation was first derived in [10] in the study of higher co-dimension mean curvature flows.

\section{Proof of Theorem}

Let $\Sigma$ be a complete submanifold of $\mathbb{R}^{2 n}$. Around any point $p \in \Sigma$, we choose orthonormal frames $\left\{e_{i}\right\}_{i=1 \cdots n}$ for $T \Sigma$ and $\left\{e_{\alpha}\right\}_{\alpha=n+1, \cdots, 2 n}$ for $N \Sigma$, the normal bundle of $\Sigma$. The convention that $i, j, k, \cdots$ denote tangent indexes and $\alpha, \beta, \gamma \cdots$ denote normal indexes is followed.

The second fundamental form of $\Sigma$ is denoted by $h_{\alpha i j}=\left\langle\nabla_{e_{i}} e_{j}, e_{\alpha}\right\rangle$.

The following formula was essentially derived in [10]. To apply to the current situation, we note that a minimal submanifold corresponds to a stationary phase of the mean curvature flow.

Proposition 2.1. Let $\Sigma$ be the graph of $f: \mathbb{R}^{n} \mapsto \mathbb{R}^{n}$ and $\left(\lambda_{i}\right)$ be the eigenvalues of $\sqrt{(d f)^{T} d f}$. If $\Sigma$ is a minimal submanifold, then $* \Omega=\frac{1}{\sqrt{\Pi_{i=1}^{n}\left(1+\lambda_{i}^{2}\right)}}$ satisfies the following equation.

$\Delta * \Omega=-* \Omega\left\{\sum_{\alpha, i, k} h_{\alpha i k}^{2}-2 \sum_{k, i<j} \lambda_{i} \lambda_{j} h_{n+i, i k} h_{n+j, j k}+2 \sum_{k, i<j} \lambda_{i} \lambda_{j} h_{n+j, i k} h_{n+i, j k}\right\}$

where $\Delta$ is the Laplace operator of the induced metric on $\Sigma$.

Geometrically, $* \Omega$ is the Jacobian of the projection from $\Sigma$ to the domain $\mathbb{R}^{n}$. The second fundamental form $h_{\alpha i j}$ are with respect to special orthonormal frames $\left\{e_{i}\right\}$ and $\left\{e_{\alpha}\right\}$, see $[10]$ for detail.

Proof of Theorem A. First we show if the Gauss map of $\Sigma$ lies in $\Xi \cap \mathfrak{B}_{K}$, then $\Sigma$ is an affine space. The general case follows from the following observation: if $g \in U(n)$ then $g(\Sigma)$ is again a minimal Lagrangian submanifold.

We rewrite equation (2.1) in the Lagrangian case. Hence the tangent bundle is canonically isomorphic to the normal bundle by the complex structure $J$. We define

$$
h_{i j k}=\left\langle\nabla_{e_{i}} e_{j}, J\left(e_{k}\right)\right\rangle
$$

then $h_{i j k}$ is symmetric in $i, j$ and $k$.

The Lagrangian condition also implies $\langle d f(X), J(Y)\rangle$ is symmetric in $X, Y$. Notice that $d f$ can be identified with Hess $F$ by $J$ for any potential function $F$. We can find an orthonormal basis $\left\{a_{i}\right\}_{i=1 \cdots n}$ for the domain $\mathbb{R}^{n}$ so that $d f\left(a_{i}\right)=$ $\lambda_{i} J\left(a_{i}\right)$. Then $\left\{e_{i}=\frac{1}{\sqrt{1+\lambda_{i}^{2}}}\left(a_{i}+\lambda_{i} J\left(a_{i}\right)\right)\right\}_{i=1, \cdots, n}$ becomes an orthonormal basis for $T_{p} \Sigma$ and $\left\{J\left(e_{i}\right)\right\}_{i=1 \cdots n}$ an orthonormal basis for the normal bundle. Equation (2.1) becomes

$$
\Delta * \Omega=-* \Omega\left\{\sum_{i, j, k} h_{i j k}^{2}-2 \sum_{k, i<j} \lambda_{i} \lambda_{j} h_{i i k} h_{j j k}+2 \sum_{k, i<j} \lambda_{i} \lambda_{j} h_{j i k} h_{i j k}\right\}
$$


We shall calculate

$$
\Delta(\ln * \Omega)=\frac{* \Omega \Delta(* \Omega)-|\nabla * \Omega|^{2}}{|* \Omega|^{2}}
$$

The covariant derivative of $* \Omega$ can be calculated as in equation (3.1) of [10].

$$
(* \Omega)_{k}=-* \Omega\left(\sum_{i} \lambda_{i} h_{i i k}\right)
$$

Plug this and equation (2.2) into equation (2.3), we obtain

$$
\Delta(\ln * \Omega)=-\left\{\sum_{i, j, k} h_{i j k}^{2}+\sum_{k, i} \lambda_{i}^{2} h_{i i k}^{2}+2 \sum_{k, i<j} \lambda_{i} \lambda_{j} h_{i j k}^{2}\right\}
$$

If the Gauss map of $\Sigma$ lies in $\Xi$, by completing square it is obvious that $\Delta(\ln * \Omega) \leq 0$. Let us first assume $\Sigma$ is a minimal Lagrangian cone. It is not hard to check the equation (2.4) still holds in this case. Now $\ln * \Omega$ assumes its minimum on $\Sigma$ and the maximum principle implies $\ln * \Omega$ is a constant. The right hand side $\sum_{i, j, k} h_{i j k}^{2}+\sum_{k, i} \lambda_{i}^{2} h_{i i k}^{2}+2 \sum_{k, i<j} \lambda_{i} \lambda_{j} h_{i j k}^{2}=0$ forces $h_{i j k}=0$ for any $i, j, k$ by symmetry considerations.

For the general case, we notice the condition $\left|\lambda_{i}\right| \leq K$ means $\Sigma$ is the graph of a vector-valued function with bounded gradient. We can then apply the standard blow-down and dimension reduction to get a minimal cone and use Allard's regularity theorem to conclude $\Sigma$ is totally geodesic and thus an affine space.

If $\Sigma$ is minimal Lagrangian, so is any $g(\Sigma)$ for $g \in U(n)$. This is because $U(n)$ is contained in the isometry group of $\mathbb{C}^{n}$ and it preserves the standard Kähler form. This completes the proof of Theorem A.

Proof of Theorem B. This follows immediately from the definition of the set $\mathfrak{M}_{K}$ and equation (2.4). Because $\Sigma$ is minimal, we only need to consider trace-free $h_{i j k}$.

We remark that when $n=3$ only the bounded gradient condition is needed. This is a special case of the classical result of Barbosa [1] and Fischer-Colbrie [4] on two-dimensional minimal cones, see also [11]. When $n \geq 3$, we identify other more specific regions of the Lagrangian Grassmannian where the Bernstein-type theorem also applies.

Corollary C. The conclusion for Theorem A holds for $\Xi^{\prime} \cap \mathfrak{B}_{K}$ where $\Xi^{\prime}$ is the subset of Lagrangian Grassmannian consisting of graphs of symmetric linear transformations $L: \mathbb{R}^{n} \mapsto \mathbb{R}^{n}$ with eigenvalues $\lambda_{i} \lambda_{j} \geq c>-\frac{3}{2}$ for any $i, j$. 
Proof. We rewrite the right hand side of equation (2.4).

$$
\begin{aligned}
& -\left\{\sum_{i, j, k} h_{i j k}^{2}+\sum_{k, i} \lambda_{i}^{2} h_{i i k}^{2}+2 \sum_{k, i<j} \lambda_{i} \lambda_{j} h_{i j k}^{2}\right\} \\
= & -\left\{\sum_{i, j, k} h_{i j k}^{2}+\sum_{i} \lambda_{i}^{2} h_{i i i}^{2}+\sum_{i<k}\left(\lambda_{i}^{2}+2 \lambda_{i} \lambda_{k}\right) h_{i i k}^{2}+\sum_{i>k}\left(\lambda_{i}^{2}+2 \lambda_{i} \lambda_{k}\right) h_{i i k}^{2}\right. \\
& \left.+2 \sum_{i<j<k}\left(\lambda_{i} \lambda_{j}+\lambda_{j} \lambda_{k}+\lambda_{k} \lambda_{i}\right) h_{i j k}^{2}\right\}
\end{aligned}
$$

We can rewrite the second fundamental form as

$$
\sum_{i, j, k} h_{i j k}^{2}=\sum_{i} h_{i i i}^{2}+\sum_{i<k} 3 h_{i i k}^{2}+\sum_{i>k} 3 h_{i i k}^{2}+\sum_{i<j<k} 6 h_{i j k}^{2} .
$$

Plug equation (2.6) into (2.5), we derive

$$
\begin{aligned}
& \Delta(\ln * \Omega) \\
= & -\left\{\sum_{i}\left(1+\lambda_{i}^{2}\right) h_{i i i}^{2}+\sum_{i<k}\left(\lambda_{i}^{2}+2 \lambda_{i} \lambda_{k}+3\right) h_{i i k}^{2}+\sum_{i>k}\left(\lambda_{i}^{2}+2 \lambda_{i} \lambda_{k}+3\right) h_{i i k}^{2}\right. \\
& \left.+2 \sum_{i<j<k}\left(\lambda_{i} \lambda_{j}+\lambda_{j} \lambda_{k}+\lambda_{k} \lambda_{i}+3\right) h_{i j k}^{2}\right\}
\end{aligned}
$$

From the assumption, there exists a positive constant $\delta$ such that $\lambda_{i} \lambda_{j}>-\frac{3}{2}+$ $\delta$ for any $i \neq j$. For any pairwise distinct $i, j, k$, at least one of $\left\{\lambda_{i} \lambda_{j}, \lambda_{j} \lambda_{k}, \lambda_{k} \lambda_{i}\right\}$ is nonnegative, therefore $\lambda_{i} \lambda_{j}+\lambda_{j} \lambda_{k}+\lambda_{k} \lambda_{i}>-3+2 \delta$.

Thus there exists a positive constant $C$ such that $\Delta(\ln * \Omega) \leq-C \delta|A|^{2}$. The rest is identical to the proof of Theorem A.

In the following, we rewrite the right hand side of equation (2.7) by using the equation $H=0$. Since $\Sigma$ is minimal, the mean curvature vector $\sum_{i} h_{i i k}=0$ for each $k$, we have

$$
\begin{aligned}
& \sum_{i} \lambda_{i}^{2} h_{i i i}^{2}=\sum_{i} \lambda_{i}^{2}\left(-\sum_{j \neq i} h_{i j j}\right)^{2} \\
= & \sum_{i<j} \lambda_{i}^{2} h_{i j j}^{2}+\sum_{i>j} \lambda_{i}^{2} h_{i j j}^{2}+2 \sum_{i \neq j, i \neq l, j<l} \lambda_{i}^{2} h_{i j j} h_{i l l}
\end{aligned}
$$

Plug equation (2.8) into equation (2.7), the righthand side of (2.7) becomes

$$
\begin{aligned}
& -\left\{\sum_{i} h_{i i i}^{2}+2 \sum_{i \neq j, i \neq l, j<l} \lambda_{i}^{2} h_{i j j} h_{i l l}+\sum_{i<k}\left(\lambda_{i}^{2}+2 \lambda_{i} \lambda_{k}+\lambda_{k}^{2}+3\right) h_{i i k}^{2}+\right. \\
& \left.\sum_{i>k}\left(\lambda_{i}^{2}+2 \lambda_{i} \lambda_{k}+\lambda_{k}^{2}+3\right) h_{i i k}^{2}+2 \sum_{i<j<k}\left(\lambda_{i} \lambda_{j}+\lambda_{j} \lambda_{k}+\lambda_{k} \lambda_{i}+3\right) h_{i j k}^{2}\right\} .
\end{aligned}
$$


Therefore we have

$$
\begin{aligned}
\Delta(\ln * \Omega)= & -\left\{\sum_{i} h_{i i i}^{2}+2 \sum_{i \neq j, i \neq l, j<l} \lambda_{i}^{2} h_{i j j} h_{i l l}+\sum_{p \neq q}\left[\left(\lambda_{p}+\lambda_{q}\right)^{2}+3\right] h_{p q q}^{2}+\right. \\
& \left.+2 \sum_{i<j<k}\left(\lambda_{i} \lambda_{j}+\lambda_{j} \lambda_{k}+\lambda_{k} \lambda_{i}+3\right) h_{i j k}^{2}\right\} .
\end{aligned}
$$

When $n=3$, the condition $\lambda_{1} \lambda_{2}+\lambda_{2} \lambda_{3}+\lambda_{3} \lambda_{1} \geq-3+\delta$ implies

$$
2 \sum_{i \neq j, i \neq l, j<l} \lambda_{i}^{2} h_{i j j} h_{i l l}+\sum_{p \neq q}\left[\left(\lambda_{p}+\lambda_{q}\right)^{2}+3\right] h_{p q q}^{2} \geq \delta \sum_{p \neq q} h_{p q q}^{2} .
$$

Therefore the Bernstein type result holds under this condition.

Remark. After this paper was finished, we were informed by Yu Yuan that he has also obtained Bernstein type results in his paper "A Bernstein problem for special Lagrangian equations" [12]. In the following, we compare our results with his.

1. We consider a vector valued function $f: \mathbb{R}^{n} \mapsto \mathbb{R}^{n}$ satisfying the minimal surface system

$$
g^{i j} \frac{\partial^{2} f^{\alpha}}{\partial x^{i} \partial x^{j}}=0
$$

where $g^{i j}$ is the inverse matrix to $\delta_{i j}+\frac{\partial f^{\alpha}}{\partial x^{i}} \frac{\partial f^{\alpha}}{\partial x^{j}}$. The Lagrangian condition implies the linear map $d f: \mathbb{R}^{n} \mapsto \mathbb{R}^{n}$ is symmetric. Yu Yuan considers a scalar function $u: \mathbb{R}^{n} \mapsto \mathbb{R}$ satisfying equation (1.1). The relation between these two formulations is $f=\nabla u$ and $d f=D^{2} u$.

2. Yu Yuan obtains an equivalent form to our formula (2.4) in [12]. This is the key formula in both his paper and ours. The derivations are nevertheless quite different. The equation satisfied by the quantity $\ln \sqrt{\operatorname{det}\left(\delta_{i j}+\frac{\partial f^{\alpha}}{\partial x^{i}} \frac{\partial f^{\alpha}}{\partial x^{j}}\right)}$ for a solution to the general minimal surface system (2.10) was essentially calculated by the second author in [10] (see equation (3.8) in [10]). The second fundamental form $h_{\alpha i j}$ becomes a symmetric three tensor $h_{k i j}$ by the Lagrangian condition. Yu Yuan derives his formula by considering $\ln \sqrt{\operatorname{det}\left(I+\left(D^{2} u\right)^{2}\right)}$ for a scalar solution $u$ to (1.1). At any given point by choosing a particular orthonormal coordinate the third derivative of $u$, $u_{i j k}$ is the same as our $h_{i j k}$.

3. Though the key formulae used in both papers are the same, the presentations of the results are different. We adopt a more geometric point of view and express them in terms of the Gauss maps. Our condition is stated as a region in the Lagrangian Grassmannian and the orbits of the region under the $U(n)$ action. Yu Yuan states his result in terms of the potential $u$ and its second derivative. Note such representation of a Lagrangian submanifold involves a choice of a base Lagrangian subspace in $\mathbb{C}^{n}$ upon which the potential function $u$ is defined and this choice is not canonical. Yu Yuan 
had the following interesting observation: the linear transformation on $\mathbb{C}^{n}$, $\left(x^{i}, y^{i}\right) \mapsto\left(\frac{x^{i}+y^{i}}{\sqrt{2}}, \frac{-x^{i}+y^{i}}{\sqrt{2}}\right), i=1 \cdots n$, takes a convex potential function $u$ to another function $\bar{u}$ (defined on a different Lagrangian subspace though) with $-I \leq D^{2} \bar{u} \leq I$. From this, we see the convexity of the potential really depends on the choice of the base Lagrangian subspace. This implies a convex entire solution to equation (1.1) is a quadratic polynomial. Since this transformation (so called Lewy transformation) is an element of $U(n)$, the implication is also contained in our Theorem A.

\section{Acknowledgements}

We wish to thank Professor D. H. Phong and Professor S.-T. Yau for their encouragement and support. We would also like to thank Yu Yuan for sending us his preprint before publication.

\section{References}

[1] J. L. M. Barbosa, An extrinsic rigidity theorem for minimal immersions from $S^{2}$ into $S^{n}$, J. Differential Geom. 14 (1979), 355-368 (1980).

[2] K. Ecker, G. Huisken, A Bernstein result for minimal graphs of controlled growth, J. Differential Geom. 31 (1990), 397-400.

[3] D. Fischer-Colbrie, Some rigidity theorems for minimal submanifolds of the sphere, Acta Math. 145 (1980), 29-46.

[4] L. Fu, An analogue of Bernstein's theorem, Houston J. Math. 24 (1998), 415-419.

[5] S. Hildebrandt, J. Jost, K.-O Widman, Harmonic mappings and minimal submanifolds, Invent. Math. 62 (1980/81), 269-298.

[6] R. Harvey, H. B. Lawson, Calibrated geometries, Acta Math. 148 (1982), 47-157.

[7] J. Jost, Y.L. Xin, Bernstein type theorems for higher codimension, Calc. Var. Partial Differential Equations 9 (1999), 277-296.

[8] _ A Bernstein theorem for special Lagrangian graphs, preprint, 2001.

[9] A. Strominger, S.-T. Yau, E. Zaslow, Mirror symmetry is T-duality, Nuclear Phys. B 479 (1996), 243-259.

[10] M-T. Wang, Long-time existence and convergence of graphic mean curvature flow in arbitrary codimension, Invent. Math. 148 (2002), 525-543.

[11] _ On graphic Berstein type results in higher codimensions, to appear in Trans. Amer. Math. Soc.

[12] Y. Yuan, A Bernstein problem for special Lagrangian equation, preprint, 2001.

Department of Mathematics, Columbia University, New York, NY 10027, U.S.A.

E-mail address: tsui@math.columbia.edu

E-mail address: mtwang@math.columbia.edu 